\title{
The distribution of real-valued $Q$-additive functions modulo 1
}

\author{
by
}

\author{
Abigail Hoit (Elmhurst, IL)
}

0. Introduction. Let $q \geq 2$ be an integer. A $q$-additive function $f$ : $\mathbb{N} \rightarrow \mathbb{C}$ is a function of the form $f(n)=\sum_{j \geq 0} f_{j}\left(a_{j}(n)\right)$ where $n=$ $\sum_{j \geq 0} a_{j}(n) q^{j}$ is the base- $q$ representation of $n$ and the "component functions" $f_{j}$ are functions defined on $\{0,1, \ldots, q-1\}$ and satisfying $f_{j}(0)=0$. These functions were introduced by A. O. Gel'fond [Ge] in 1968, and have been studied by Coquet [Co1], Delange [De3], and others. They generalize the sum-of-digits functions $s_{q}(n)$ with respect to base $q$.

In 1977, Coquet [Co1] generalized $q$-additive functions to more general systems of numeration. Specifically, he considered so-called mixed radix representations (also called Cantor representations) defined as follows. Let $Q=\left\{Q_{j}\right\}_{j \geq 0}$ be a sequence of strictly increasing positive integers with $Q_{0}=1$ such that $Q_{j} \mid Q_{j+1}$ for all $j$. Note that the sequence $Q$ is uniquely determined by the factors $q_{j}=Q_{j+1} / Q_{j}$. It is easily seen that each nonnegative integer $n$ has a unique "base- $Q$ " representation of the form $n=$ $\sum_{j \geq 0} a_{j}(n) Q_{j}$, where the "digits" $a_{j}(n)$ satisfy $0 \leq a_{j}(n)<q_{j}$. Examples of such representations are the ordinary base- $q$ representations $\left(q_{j}=q\right)$ as well as the factorial representation $\left(q_{j}=j+2\right)$, the factorial-squared representation $\left(q_{j}=(j+2)^{2}\right)$, and the doubly-geometric representations $\left(q_{j}=q^{j}\right)$. For a full discussion of these and other numeration systems see, for example, Grabner et al. [GLT] or the survey article by Fraenkel [Fr] and the references therein.

Given a mixed radix system $Q$, Coquet defined a $Q$-additive function $f: \mathbb{N} \rightarrow \mathbb{C}$ to be a function of the form $f(n)=\sum_{j \geq 0} f_{j}\left(a_{j}(n)\right)$ where $n=$ $\sum_{j \geq 0} a_{j}(n) Q_{j}$ is the base- $Q$ representation of $n$ and the component functions $f_{j}$ are functions defined on $\left\{0,1, \ldots, q_{j}-1\right\}$ and satisfying $f_{j}(0)=0$.

2000 Mathematics Subject Classification: Primary 11K16.

The results in this paper also appear in the author's doctoral thesis deposited in April of 1999 at the University of Illinois at Urbana-Champaign. 
A simple example of a $Q$-additive function is the sum-of-digits function $s_{Q}(n)=\sum_{j \geq 0} a_{j}(n)$, which corresponds to the choice $f_{j}(a)=a$. This function has been studied by Kirschenhofer and Tichy [KT], among others. For recent work on general $Q$-additive functions see Manstavičius [Ma]. For generalizations of $q$-additive functions to other numeration systems see, for example, Barat and Grabner [BG].

Our main result, Theorem 1, characterizes those real-valued $Q$-additive functions that have a limit distribution (resp. uniform limit distribution) modulo 1 . In order to prove this result, we consider so-called $Q$-multiplicative functions, which are defined in analogy to $Q$-additive functions as follows. A $Q$-multiplicative function is a function $g: \mathbb{N} \rightarrow \mathbb{C}$ of the form $g(n)=$ $\prod_{j \geq 0} g_{j}\left(a_{j}(n)\right)$, where $n=\sum_{j \geq 0} a_{j}(n) Q_{j}$ is the base- $Q$ representation of $n$ and the component functions $g_{j}$ are functions defined on $\left\{0,1, \ldots, q_{j}-1\right\}$ and satisfying $g_{j}(0)=1$. These functions have been studied by Coquet [Co1] and others, usually in conjunction with work on $Q$-additive functions. We establish mean value theorems for $Q$-multiplicative functions analogous to those of Delange and Wirsing (see, e.g., [El, Chapter 6]) for ordinary multiplicative functions.

Throughout this paper, we set $e(x)=e^{2 \pi i x}$ and write $\|x\|$ to denote the distance from $x$ to the nearest integer and $\{x\}$ to denote the fractional part of $x$.

1. Statement of results. Let $Q=\left\{Q_{j}\right\}_{j \geq 0}$ be a mixed radix system with factors $q_{j}=Q_{j+1} / Q_{j}$. Let $f$ be a real-valued $Q$-additive function with component functions $f_{j}$. We say that $f$ has a limit distribution modulo 1 if there is a distribution function $F$ (i.e., $F$ is right-continuous and monotonic with $F(x)=0$ for $x<0$ and $F(x)=1$ for $x \geq 1$ ) such that the limit

$$
\lim _{N \rightarrow \infty} \frac{1}{N} \#\{0 \leq n<N:\{f(n)\} \leq x\}
$$

exists and equals $F(x)$ for every $x$ at which $F$ is continuous. We say that $f$ has a uniform limit distribution modulo 1 if this holds with $F(x)=x$ for $0 \leq x<1$. Aside from its intrinsic interest, the study of the distribution modulo 1 of $Q$-additive functions is motivated in part by the results of Coquet [Co1] and Mendès France $[\mathrm{MF}]$ connecting the uniform distribution of certain $Q$-additive functions to so-called P-V numbers. Our main result is a complete characterization of real-valued $Q$-additive functions that have a limit distribution (resp. uniform limit distribution) modulo 1.

THEOREM 1. A real-valued $Q$-additive function $f$ has a limit distribution modulo 1 if and only if, for each integer $k \neq 0$, at least one of the following conditions holds:

(i) There exists $j \geq 0$ such that $\sum_{0 \leq n<q_{j}} e\left(k f_{j}(n)\right)=0$. 
(ii) The series

$$
\sum_{j \geq 0}\left(1-\frac{1}{q_{j}}\left|\sum_{0 \leq n<q_{j}} e\left(k f_{j}(n)\right)\right|\right)
$$

diverges.

(iii) The series

$$
\sum_{j \geq 0}\left(1-\frac{1}{q_{j}} \sum_{0 \leq n<q_{j}} e\left(k f_{j}(n)\right)\right)
$$

converges, and

$$
\lim _{j \rightarrow \infty}\left(\max _{0<n \leq q_{j}} \frac{1}{n} \sum_{0 \leq m<n}\left\|k f_{j}(m)\right\|^{2}\right)=0 .
$$

Furthermore, $f$ is uniformly distributed modulo 1 if and only if, for all integers $k \neq 0$, at least one of conditions (i) or (ii) holds.

This result generalizes the characterization given by Kim [Ki, p. 27] for the special case of $q$-additive functions.

We apply Theorem 1 to derive several corollaries that deal with special cases. We first consider numeration systems in which the factors $q_{j}$ are bounded. In particular, these systems include the ordinary base- $q$ representations generated by $q_{j}=q$ for all $j$.

COROLlary 1. Suppose the factors $q_{j}$ are bounded. Then $f$ is uniformly distributed modulo 1 if and only if, for all $k \neq 0$, either the series

$$
\sum_{j \geq 0} \sum_{0 \leq n<q_{j}}\left\|k f_{j}(n)\right\|^{2}
$$

diverges, or for some $j \geq 0$ we have

$$
\sum_{0 \leq n<q_{j}} e\left(k f_{j}(n)\right)=0 .
$$

Let $\alpha \in \mathbb{R}$. We call an integer-valued arithmetic function $f$ normal if the function $\alpha f$ is uniformly distributed modulo 1 if and only if $\alpha$ is irrational. Coquet [Co2] showed that for any base $q \geq 2$, the associated sum-of-digits function $s_{q}(n)$ is normal. General criteria for the normality of arithmetic functions have been given by Drmota and Tichy [DT, Section 1.4.3]. In Corollaries 2 and 3 below, we apply Theorem 1 to show that two classes of integer-valued $Q$-additive functions are normal.

Corollary 2. For any mixed radix numeration system $Q$, the function $s_{Q}(n)$, the sum of digits in the base- $Q$ representation of $n$, is normal.

We call a $Q$-additive function $f$ completely $Q$-additive if there exists a function $g: \mathbb{N} \rightarrow \mathbb{C}$ such that, for all $j \geq 0$ and $0 \leq n<q_{j}, f_{j}(n)=g(n)$, 
i.e., if the component functions $f_{j}$ are independent of $j$ on their respective domains. The following corollary generalizes a result of Drmota and Tichy [DT, Theorem 1.99].

Corollary 3. Suppose that the series $\sum_{j \geq 0} 1 / q_{j}$ diverges. Let $f$ be a completely $Q$-additive, integer-valued function such that there is some integer $1 \leq a<\min _{j} q_{j}$ with $f_{0}(a)>0$. Then $f$ is normal.

In the next two corollaries we investigate the normality of two particular integer-valued $Q$-additive functions that have been previously considered in [Ho, examples (c) and (a)] and [KT, Theorem 3]. These results provide examples of functions that have a non-uniform limit distribution modulo 1 as well as functions that have no limit distribution modulo 1 .

Corollary 4. Let $\alpha$ be an irrational number. Let $M(n)$ be the number of digits in the base- $Q$ representation of $n$ which are maximal, and set $f(n)=\alpha M(n)$. Then $f$ has a limit distribution modulo 1 . Moreover, the limit distribution is uniform if and only if the series $\sum_{j \geq 0} 1 / q_{j}$ diverges.

Corollary 5. Let $a>0$ be a fixed integer and let $\alpha$ be an irrational number. Let $N_{a}(n)$ be the number of digits a in the base-Q representation of $n$, and set $f(n)=\alpha N_{a}(n)$. Then $f$ is uniformly distributed modulo 1 if and only if $\sum_{q_{j}>a} 1 / q_{j}$ diverges. The function $f$ has a non-uniform limit distribution modulo 1 if and only if $q_{j} \leq$ a for all but at most finitely many $j$.

2. Lemmas. The first lemma is a well known result on the distribution modulo 1 of real-valued arithmetic functions (see, e.g., [De2, p. 216]). The second assertion of the lemma is known as Weyl's Criterion [We].

Lemma 1. A real-valued arithmetic function $f$ has a limit distribution modulo 1 if and only if, for each integer $k \neq 0$,

$$
\lim _{N \rightarrow \infty} \frac{1}{N} \sum_{n=0}^{N-1} e(k f(n))
$$

exists. Further, the distribution is uniform modulo 1 if and only if, for each integer $k \neq 0$, the above limit is 0 .

Throughout the remainder of this section, we fix a mixed radix system $Q$ with factors $\left\{q_{j}\right\}_{j \geq 0}$. We denote by $g$ a $Q$-multiplicative function satisfying $|g| \leq 1$ with component functions $g_{j}$, and define

$$
\mu_{j}(g)=\frac{1}{q_{j}} \sum_{0 \leq n<q_{j}} g_{j}(n) .
$$

Thus, $\mu_{j}(g)$ is the mean value of $g_{j}$ on its domain $\left\{0,1, \ldots, q_{j}-1\right\}$.

The following lemma relates the mean value of $g$ on $\left\{0,1, \ldots, r Q_{j}-1\right\}$ to that of the functions $g_{i}$. 
Lemma 2. For $j \geq 0$ and any positive integer $r$ with $1 \leq r \leq q_{j}$ we have

$$
\frac{1}{r Q_{j}} \sum_{n=0}^{r Q_{j}-1} g(n)=\left(\frac{1}{r} \sum_{n=0}^{r-1} g_{j}(n)\right)\left(\frac{1}{Q_{j}} \sum_{n=0}^{Q_{j}-1} g(n)\right) .
$$

Moreover, for any $j \geq 0$,

$$
\frac{1}{Q_{j}} \sum_{n=0}^{Q_{j}-1} g(n)=\prod_{i=0}^{j-1} \mu_{i}(g)
$$

Proof. We first note that, since $Q_{0}=1$ and $g(0)=1$, we have

$$
\frac{1}{Q_{0}} \sum_{n=0}^{Q_{0}-1} g(n)=g(0)=1
$$

Thus, relation (2.2) follows from (2.1) by applying (2.1) with $r=q_{j}$ and iterating the identity. Hence it suffices to prove $(2.1)$.

Observe that any non-negative integer $n<r Q_{j}$ can be written uniquely (via the division algorithm) in the form $n=s Q_{j}+t$ with $0 \leq t<Q_{j}$ and $0 \leq s<r$. By the $Q$-multiplicativity of $g$, we have, with this representation,

$$
g(n)=g_{j}(s) g(t)
$$

As $n$ runs through the set $\left\{0,1, \ldots, r Q_{j}-1\right\}, s$ and $t$ run independently through the sets $\{0,1, \ldots, r-1\}$ and $\left\{0,1, \ldots, Q_{j}-1\right\}$, respectively. It follows that

$$
\frac{1}{r Q_{j}} \sum_{n=0}^{r Q_{j}-1} g(n)=\frac{1}{r Q_{j}} \sum_{s=0}^{r-1} \sum_{t=0}^{Q_{j}-1} g_{j}(s) g(t)=\frac{1}{r} \sum_{s=0}^{r-1} g_{j}(s) \frac{1}{Q_{j}} \sum_{t=0}^{Q_{j}-1} g(t),
$$

which is (2.1).

To obtain necessary and sufficient conditions for the convergence of the product in (2.2), we will use the following lemma, a proof of which can be found in many elementary texts on complex variables (see, e.g., [LR, pp. 383-384]).

Lemma 3. Let $z_{0}, z_{1}, \ldots$ be complex numbers satisfying $\left|z_{j}\right| \leq 1$, and let $P_{i}=\prod_{j=0}^{i} z_{j}$. Then $\lim _{i \rightarrow \infty} P_{i}=0$ if and only if at least one of the following two conditions holds:

(i) There is some $j \geq 0$ such that $z_{j}=0$.

(ii) $\sum_{j=0}^{\infty}\left(1-\left|z_{j}\right|\right)=\infty$.

Then $\lim _{i \rightarrow \infty} P_{i}$ exists and is non-zero if and only if the following two conditions are both satisfied:

(iii) $z_{j} \neq 0$ for all $j$.

(iv) $\sum_{j=0}^{\infty}\left(1-z_{j}\right)$ converges. 
The next lemma relates the mean value of $g$ on $\{0,1, \ldots, N-1\}$ for general integers $N$ to the mean values of the functions $g_{j}$.

Lemma 4. Let $N$ be a positive integer and let $\sum_{j=0}^{i} a_{j} Q_{j}$, with $a_{i}>0$, be the base- $Q$ representation of $N$. Then

$$
\begin{aligned}
& \frac{1}{N} \sum_{n=0}^{N-1} g(n) \\
& \quad=\sum_{j=0}^{i} \frac{a_{j} Q_{j}}{N}\left(\prod_{m=j+1}^{i} g_{m}\left(a_{m}\right)\right)\left(\frac{1}{a_{j}} \sum_{n=0}^{a_{j}-1} g_{j}(n)\right)\left(\prod_{k=0}^{j-1} \mu_{k}(g)\right),
\end{aligned}
$$

where empty products and empty sums are to be interpreted as 1 and 0 , respectively. Furthermore, for any positive integer $h \leq i$, we have

$$
\sum_{j=0}^{i-h} \frac{a_{j} Q_{j}}{N}<2^{1-h} .
$$

Proof. We begin by dividing the interval $0 \leq n<N$ into the subintervals $0 \leq n<a_{i} Q_{i}$ and $a_{i} Q_{i} \leq n<N$, to obtain

$$
\frac{1}{N} \sum_{n=0}^{N-1} g(n)=\frac{1}{N} \sum_{0 \leq n<a_{i} Q_{i}} g(n)+\frac{1}{N} \sum_{a_{i} Q_{i} \leq n<N} g(n) .
$$

We have, by Lemma 2 ,

$$
\begin{aligned}
\frac{1}{N} \sum_{0 \leq n<a_{i} Q_{i}} g(n) & =\frac{a_{i} Q_{i}}{N}\left(\frac{1}{a_{i}} \sum_{n=0}^{a_{i}-1} g_{i}(n)\right)\left(\frac{1}{Q_{i}} \sum_{n=0}^{Q_{i}-1} g(n)\right) \\
& =\frac{a_{i} Q_{i}}{N}\left(\frac{1}{a_{i}} \sum_{n=0}^{a_{i}-1} g_{i}(n)\right)\left(\prod_{k=0}^{i-1} \mu_{k}(g)\right) .
\end{aligned}
$$

Furthermore, by the $Q$-multiplicativity of $g$, we also have, for all $n$ with $a_{i} Q_{i} \leq n<N, g(n)=g\left(n-a_{i} Q_{i}\right) g_{i}\left(a_{i}\right)$. Thus,

$$
\begin{aligned}
\frac{1}{N} \sum_{a_{i} Q_{i} \leq n<N} g(n) & =\frac{1}{N} \sum_{a_{i} Q_{i} \leq n<N} g\left(n-a_{i} Q_{i}\right) g_{i}\left(a_{i}\right) \\
& =g_{i}\left(a_{i}\right) \frac{1}{N} \sum_{a_{i} Q_{i} \leq n<N} g\left(n-a_{i} Q_{i}\right) \\
& =g_{i}\left(a_{i}\right) \frac{1}{N} \sum_{0 \leq n<N-a_{i} Q_{i}} g(n) \\
& =g_{i}\left(a_{i}\right) \frac{N-a_{i} Q_{i}}{N}\left(\frac{1}{N-a_{i} Q_{i}} \sum_{0 \leq n<N-a_{i} Q_{i}} g(n)\right) .
\end{aligned}
$$


It follows that

$$
\begin{aligned}
\frac{1}{N} \sum_{n=0}^{N-1} g(n)= & \frac{a_{i} Q_{i}}{N}\left(\frac{1}{a_{i}} \sum_{n=0}^{a_{i}-1} g_{i}(n)\right)\left(\prod_{k=0}^{i-1} \mu_{k}(g)\right) \\
& +g_{i}\left(a_{i}\right) \frac{N-a_{i} Q_{i}}{N}\left(\frac{1}{N-a_{i} Q_{i}} \sum_{0 \leq n<N-a_{i} Q_{i}} g(n)\right) .
\end{aligned}
$$

Iterating the last expression $i-1$ times gives (2.3). Inequality (2.4) follows from the chain of inequalities

$$
\frac{1}{N} \sum_{j=0}^{i-h} a_{j} Q_{j} \leq \frac{1}{Q_{i}} \sum_{j=0}^{i-h} a_{j} Q_{j} \leq \frac{Q_{i-h+1}}{Q_{i}}=\prod_{j=i-h+1}^{i-1} \frac{1}{q_{j}} \leq 2^{1-h} .
$$

3. Mean value theorems for $Q$-multiplicative functions. Throughout this section, we let $Q$ be a mixed radix system with factors $\left\{q_{j}\right\}_{j \geq 0}$. For a given $Q$-multiplicative function $g$ with component functions $g_{j}$, we define the mean value of $g$ by

$$
M(g)=\lim _{N \rightarrow \infty} \frac{1}{N} \sum_{0 \leq n<N} g(n),
$$

provided this limit exists. We set

$$
\sigma_{j}(g)=\max _{0<n \leq q_{j}} \frac{1}{n} \sum_{0 \leq m<n}\left(1-\operatorname{Re}\left(g_{j}(m)\right)\right),
$$

and recall the notation

$$
\mu_{j}(g)=\frac{1}{q_{j}} \sum_{0 \leq n<q_{j}} g_{j}(n)
$$

introduced in the previous section.

The following theorem, due to Coquet [Co1, Lemma 1], gives a characterization of $Q$-multiplicative functions of modulus at most 1 which have mean value 0 . We present a proof here for completeness.

TheOREM 2. Let $g$ be a $Q$-multiplicative function satisfying $|g| \leq 1$. The mean value $M(g)$ exists and is equal to 0 if and only if at least one of the following two conditions holds:

(i) For some $j \geq 0, \mu_{j}(g)=0$.

(ii) The series $\sum_{j \geq 0}\left(1-\left|\mu_{j}(g)\right|\right)$ diverges.

Proof. Assume first that $M(g)=0$. Then, by (2.2) of Lemma 2,

$$
\prod_{i=0}^{\infty} \mu_{i}(g)=\lim _{j \rightarrow \infty} \prod_{i=0}^{j} \mu_{i}(g)=\lim _{j \rightarrow \infty} \frac{1}{Q_{j+1}} \sum_{n=0}^{Q_{j+1}-1} g(n)=0 .
$$

By Lemma 3, this implies that at least one of conditions (i) or (ii) holds. 
Conversely, assume that at least one of conditions (i) or (ii) holds. Applying Lemma 3 again, we conclude that $\prod_{j=0}^{\infty} \mu_{j}(g)=0$. We now show that $M(g)$ exists and is equal to 0 . Let $N$ be a positive integer with base- $Q$ representation $\sum_{j=0}^{i} a_{j} Q_{j}$, where $a_{i}>0$. Applying Lemma 4 with $h=\lfloor i / 2\rfloor$, we obtain

$$
\begin{aligned}
\mid \frac{1}{N} & \sum_{0 \leq n<N} g(n) \mid \\
& <\sum_{j=0}^{i} \frac{a_{j} Q_{j}}{N}\left|\prod_{k=0}^{j-1} \mu_{k}(g)\right|<2^{1-\lfloor i / 2\rfloor}+\sum_{j=i-\lfloor i / 2\rfloor+1}^{i} \frac{a_{j} Q_{j}}{N}\left|\prod_{k=0}^{j-1} \mu_{k}(g)\right| \\
& \leq 2^{2-i / 2}+\sum_{j=\lfloor i / 2\rfloor}^{i} \frac{a_{j} Q_{j}}{N}\left|\prod_{k=0}^{j-1} \mu_{k}(g)\right| .
\end{aligned}
$$

Since $i$ tends to infinity as $N$ tends to infinity and $\sum_{j=\lfloor i / 2\rfloor}^{i} a_{j} Q_{j} \leq N$, the right-hand side tends to 0 as $N$ tends to infinity. Thus, $M(g)=0$. This completes the proof of Theorem 2 .

We now characterize those $Q$-multiplicative functions of modulus at most 1 having a non-zero mean value, a case that was not considered by Coquet. This characterization is the content of the following theorem which represents an analog of the well known mean value theorem of Delange [De1], and generalizes a result of Delange [De3] for the case of ordinary base- $q$ expansions.

TheOREM 3. Let $g$ be a $Q$-multiplicative function satisfying $|g| \leq 1$. The mean value $M(g)$ exists and is non-zero if and only if the following three conditions all hold:

(i) For each $j \geq 0, \mu_{j}(g) \neq 0$.

(ii) $\sum_{j \geq 0}\left(1-\mu_{j}(g)\right)$ converges.

(iii) $\lim _{j \rightarrow \infty} \sigma_{j}(g)=0$.

Proof. For simplicity of notation, we will write $\mu_{j}=\mu_{j}(g)$ and $\sigma_{j}=$ $\sigma_{j}(g)$ throughout the proof.

Assume first that $M(g)=L$ for some number $L \neq 0$. Then, in particular, we have

$$
\lim _{j \rightarrow \infty} \frac{1}{Q_{j}} \sum_{0 \leq n<Q_{j}} g(n)=L .
$$

By (2.2) of Lemma 2, this implies that $\prod_{j=0}^{\infty} \mu_{j}=L$. By Lemma 3, the convergence of the product $\prod_{j=0}^{\infty} \mu_{j}$ to a non-zero value implies that conditions (i) and (ii) of the theorem hold. 
It remains to show that condition (iii) also holds, i.e., we wish to show that the quantity

$$
\sigma_{j}=\max _{0<n \leq q_{j}} \frac{1}{n} \sum_{0 \leq m<n}\left(1-\operatorname{Re}\left(g_{j}(m)\right)\right)
$$

tends to zero as $j$ tends to infinity. Let $\left\{n_{j}\right\}_{j=0}^{\infty}$ be a sequence of integers such that the maximum in the definition of $\sigma_{j}$ is attained at $n=n_{j}$, so that

$$
\sigma_{j}=1-\operatorname{Re}\left(\frac{1}{n_{j}} \sum_{0 \leq m<n_{j}} g_{j}(m)\right) .
$$

Applying (2.1) of Lemma 2 with $r=n_{j}$, we obtain

$$
\lim _{j \rightarrow \infty} \frac{1}{n_{j}} \sum_{0 \leq m<n_{j}} g_{j}(m)=\lim _{j \rightarrow \infty} \frac{\left(1 / n_{j} Q_{j}\right) \sum_{0 \leq n<n_{j} Q_{j}} g(n)}{\left(1 / Q_{j}\right) \sum_{0 \leq n<Q_{j}} g(n)}=\frac{L}{L}=1 .
$$

Therefore, $\sigma_{j}$ tends to 0 as $j$ tends to infinity, which proves condition (iii).

Conversely, assume that conditions (i), (ii), and (iii) all hold. The first two conditions imply that the infinite product $\prod_{j=0}^{\infty} \mu_{j}$ converges to a nonzero value, by Lemma 3 . Let $L$ denote this value. We will show that $M(g)$ exists and is equal to $L$.

We first note that, by the bound $\left|g_{j}\right| \leq 1$ and the general inequality

$$
|1-z|^{2}=1+|z|^{2}-2 \operatorname{Re} z \leq 2(1-\operatorname{Re} z) \quad(|z| \leq 1),
$$

condition (iii) is equivalent to

$$
\lim _{j \rightarrow \infty} \max _{0<n \leq q_{j}}\left|1-\frac{1}{n} \sum_{0 \leq m<n} g_{j}(m)\right|=0 .
$$

Furthermore, (iii)' implies that

$$
\lim _{j \rightarrow \infty} g_{j}(m)=1
$$

for any fixed $m$.

Let $\varepsilon>0$ be given and choose $h$ and $i_{0}$ such that $2^{1-h}<\varepsilon$, and for $i \geq i_{0}$ we have the following three conditions:

(a) $\left|\prod_{j=0}^{i-1} \mu_{j}-L\right|<\varepsilon$.

(b) $\left|(1 / n) \sum_{0 \leq m<n} g_{i}(m)-1\right|<\varepsilon \quad\left(0<n \leq q_{i}\right)$.

(c) $\left|\prod_{k<j \leq i} g_{j}\left(m_{j}\right)-1\right|<\varepsilon \quad\left(i-h \leq k \leq i, 0 \leq m_{j}<1 / \varepsilon\right)$.

Condition (a) is possible since $\prod_{j=0}^{\infty} \mu_{j}=L$, while conditions (b) and (c) can be met in view of condition (iii) ${ }^{\prime}$ and (3.1). 
Let $N$ be a positive integer with base- $Q$ representation $N=\sum_{j=0}^{i} a_{j} Q_{j}$ where $a_{i}>0$, and suppose that $N$ is sufficiently large and $i>i_{0}+h$. Applying Lemma 4, we have

$$
\begin{aligned}
\mid \frac{1}{N} & \sum_{0 \leq n<N} g(n)-L \mid \\
= & \left|\sum_{j=0}^{i} \frac{a_{j} Q_{j}}{N}\left(\left(\prod_{m=j+1}^{i} g_{m}\left(a_{m}\right)\right)\left(\frac{1}{a_{j}} \sum_{0 \leq n<a_{j}} g_{j}(n)\right)\left(\prod_{k=0}^{j-1} \mu_{k}\right)-L\right)\right| \\
\leq & \left|\sum_{j=i-h+1}^{i} \frac{a_{j} Q_{j}}{N}\left(\left(\prod_{m=j+1}^{i} g_{m}\left(a_{m}\right)\right)\left(\frac{1}{a_{j}} \sum_{0 \leq n<a_{j}} g_{j}(n)\right)\left(\prod_{k=0}^{j-1} \mu_{k}\right)-L\right)\right| \\
& +2 \sum_{j=0}^{i-h} \frac{a_{j} Q_{j}}{N},
\end{aligned}
$$

where in the last step we have used the fact that $g_{m}, g, \mu_{k}$, and $L$ are at most 1 in modulus. By inequality (2.4) of Lemma 4, the second term on the right hand side is at most $2\left(2^{1-h}\right)<2 \varepsilon$. Moreover, by the triangle inequality, the first term is bounded by

$$
\begin{aligned}
& \sum_{j=i-h+1}^{i} \frac{a_{j} Q_{j}}{N}\left|\left(\prod_{m=j+1}^{i} g_{m}\left(a_{m}\right)\right)-1\right| \cdot\left|\left(\frac{1}{a_{j}} \sum_{0 \leq n<a_{j}} g_{j}(n)\right)\left(\prod_{k=0}^{j-1} \mu_{k}\right)\right| \\
& \quad+\sum_{j=i-h+1}^{i} \frac{a_{j} Q_{j}}{N}\left(\left|\frac{1}{a_{j}} \sum_{0 \leq n<a_{j}} g_{j}(n)-1\right| \cdot\left|\prod_{k=0}^{j-1} \mu_{k}\right|+\left|\prod_{k=0}^{j-1} \mu_{k}-L\right|\right) \\
& =\Sigma_{1}+\Sigma_{2},
\end{aligned}
$$

say. Since $i-h>i_{0}$, we have, by assumptions (a) and (b) above,

$$
\Sigma_{2}<2 \varepsilon \sum_{j=i-h+1}^{i} \frac{a_{j} Q_{j}}{N} \leq 2 \varepsilon
$$

To estimate $\Sigma_{1}$, we distinguish two cases. If $a_{j}<1 / \varepsilon$ for all $j$ with $i-h<j \leq i$, then by assumption $(\mathrm{c}),\left|\left(\prod_{m=j+1}^{i} g_{m}\left(a_{m}\right)\right)-1\right|<\varepsilon$ for all $j$ and therefore $\Sigma_{1}<\varepsilon$. Otherwise, let $j_{0}$ be the largest value of $j$ in the range $i-h<j \leq i$ for which $a_{j_{0}} \geq 1 / \varepsilon$. The contribution of terms with $j_{0} \leq j \leq i$ to $\Sigma_{1}$ is, as before, at most $\varepsilon$. Thus,

$$
\Sigma_{1}<\varepsilon+\sum_{j=i-h+1}^{j_{0}-1} \frac{a_{j} Q_{j}}{N}\left|\left(\prod_{m=j+1}^{i} g_{m}\left(a_{m}\right)\right)-1\right| \cdot\left|\left(\frac{1}{a_{j}} \sum_{0 \leq n<a_{j}} g(n)\right)\left(\prod_{k=0}^{j-1} \mu_{k}\right)\right|
$$




$$
\begin{aligned}
& \leq \varepsilon+2 \sum_{j=i-h+1}^{j_{0}-1} \frac{a_{j} Q_{j}}{N} \leq \varepsilon+2 \sum_{j=0}^{j_{0}-1} \frac{\left(q_{j}-1\right) Q_{j}}{N}=\varepsilon+2 \sum_{j=0}^{j_{0}-1} \frac{Q_{j+1}-Q_{j}}{N} \\
& <\varepsilon+\frac{2 Q_{j_{0}}}{N} \leq \varepsilon+\frac{2 Q_{j_{0}}}{a_{j_{0}} Q_{j_{0}}} \leq 3 \varepsilon .
\end{aligned}
$$

In either case, we have

$$
\left|\frac{1}{N} \sum_{0 \leq n<N} g(n)-L\right|<7 \varepsilon .
$$

Since $\varepsilon$ was arbitrary, we have shown that $M(g)=L$. This completes the proof of the theorem.

The following result is an immediate consequence of Theorems 2 and 3 .

TheOREM 4. Let $g$ be a $Q$-multiplicative function satisfying $|g| \leq 1$. The mean value $M(g)$ exists if and only if at least one of the following three conditions holds:

(i) For some $j \geq 0, \mu_{j}(g)=0$.

(ii) The series $\sum_{j \geq 0}\left(1-\left|\mu_{j}(g)\right|\right)$ diverges.

(iii) $\sum_{j \geq 0}\left(1-\mu_{j}(g)\right)$ converges, and $\lim _{j \rightarrow \infty} \sigma_{j}(g)=0$.

The mean value is zero if either condition (i) or (ii) holds.

4. Proof of Theorem 1. Let $Q=\left\{Q_{j}\right\}_{j \geq 0}$ be a mixed radix system, with factors $q_{j}=Q_{j+1} / Q_{j}$, and let $f$ be a real-valued $Q$-additive function with component functions $f_{j}$.

For each integer $k \neq 0$, we set $g^{(k)}(n)=e(k f(n))$. Then each function $g^{(k)}$ is a $Q$-multiplicative function with component functions $g_{j}^{(k)}(n)=$ $e\left(k f_{j}(n)\right)$. We write

$$
\mu_{j}^{(k)}=\mu_{j}\left(g^{(k)}\right)=\frac{1}{q_{j}} \sum_{0 \leq n<q_{j}} g_{j}^{(k)}(n),
$$

and

$$
\sigma_{j}^{(k)}=\sigma_{j}\left(g^{(k)}\right)=\max _{0<n \leq q_{j}} \frac{1}{n} \sum_{0 \leq m<n}\left(1-\operatorname{Re}\left(g_{j}^{(k)}(n)\right)\right),
$$

and denote the mean value of $g^{(k)}$ by $M_{k}$, whenever this mean value exists. By Lemma $1, f$ has a limit distribution modulo 1 if and only if, for each integer $k \neq 0$, the mean value $M_{k}$ exists, and the distribution is uniform if and only if, for each integer $k \neq 0, M_{k}=0$. By Theorem 4 , for each $k \neq 0$, $M_{k}$ exists if and only if at least one of the following three conditions holds:

(i) $k$ For some $j \geq 0, \mu_{j}^{(k)}=0$.

(ii) $)_{k}$ The series $\sum_{j \geq 0}\left(1-\left|\mu_{j}^{(k)}\right|\right)$ diverges. 
(iii) $\sum_{j \geq 0}\left(1-\mu_{j}^{(k)}\right)$ converges, and $\lim _{j \rightarrow \infty} \sigma_{j}^{(k)}=0$.

Further, $M_{k}=0$ if and only if either condition (i) ${ }_{k}$ or (ii) ${ }_{k}$ holds. Therefore, it remains only to show that, for each integer $k \neq 0$, conditions (i) ${ }_{k}$, (ii) $)_{k}$, and (iii) $_{k}$ are equivalent to conditions (i), (ii), and (iii) of Theorem 1, respectively.

To prove this, we fix an integer $k \neq 0$. Conditions (i) $k$ and (ii) $k$ are identical to conditions (i) and (ii) of Theorem 1, respectively, by the definition of $\mu_{j}^{(k)}$. The equivalence between condition (iii) $k$ and condition (iii) of Theorem 1 follows from the definition of $\mu_{j}^{(k)}$ and the relation

$$
\sigma_{j}^{(k)}=\max _{0<n \leq q_{j}} \frac{1}{n} \sum_{0 \leq m<n}\left(1-\operatorname{Re}\left(g_{j}^{(k)}(n)\right)\right) \asymp \max _{0<n \leq q_{j}} \frac{1}{n} \sum_{0 \leq m<n}\left\|k f_{j}(n)\right\|^{2},
$$

which holds since

$$
1-\operatorname{Re} e(x)=1-\cos (2 \pi x) \asymp\|x\|^{2}
$$

for any real number $x$. This completes the proof of Theorem 1 .

\section{Proof of the corollaries}

Proof of Corollary 1. Fix an integer $k \neq 0$. For each $j \geq 0$, let $n_{j}$ be such that $\max _{0 \leq n<q_{j}}\left\|k f_{j}(n)\right\|^{2}$ is attained at $n=n_{j}$. First we note that by the elementary inequality

$$
|1+e(x)| \leq 2-2\|x\|^{2} \quad(x \in \mathbb{R}),
$$

we have, for all $j$,

$$
\begin{aligned}
\frac{1}{q_{j}}\left|\sum_{0 \leq n<q_{j}} e\left(k f_{j}(n)\right)\right| & \leq \frac{1}{q_{j}}\left|\sum_{\substack{1 \leq n<q_{j} \\
n \neq n_{j}}} e\left(k f_{j}(n)\right)\right|+\frac{1}{q_{j}}\left|1+e\left(k f_{j}\left(n_{j}\right)\right)\right| \\
& \leq \frac{1}{q_{j}}\left(\left(q_{j}-2\right)+\left|1+e\left(k f_{j}\left(n_{j}\right)\right)\right|\right) \\
& \leq \frac{1}{q_{j}}\left(\left(q_{j}-2\right)+\left(2-2\left\|k f_{j}\left(n_{j}\right)\right\|^{2}\right)\right) \\
& =\frac{1}{q_{j}}\left(q_{j}-2\left\|k f_{j}\left(n_{j}\right)\right\|^{2}\right)
\end{aligned}
$$

and thus

$$
1-\frac{1}{q_{j}}\left|\sum_{0 \leq n<q_{j}} e\left(k f_{j}(n)\right)\right| \geq \frac{2\left\|k f_{j}\left(n_{j}\right)\right\|^{2}}{q_{j}} \geq \frac{2}{q_{j}^{2}} \sum_{0 \leq n<q_{j}}\left\|k f_{j}\left(n_{j}\right)\right\|^{2} .
$$

Since, by assumption, the factors $q_{j}$ are bounded, the divergence of the series

$$
\sum_{j \geq 0} \sum_{0 \leq n<q_{j}}\left\|k f_{j}(n)\right\|^{2}
$$


implies that condition (ii) of Theorem 1 holds. Hence, if for all $k \neq 0$ either the series in (5.1) diverges or condition (i) of Theorem 1 holds, then Theorem 1 implies that $f$ has a uniform limit distribution modulo 1.

Conversely, assume that $f$ is uniformly distributed modulo 1 . Then, for each $k \neq 0$, either condition (i) or condition (ii) of Theorem 1 holds. We will show that if condition (ii) holds for some $k \neq 0$ then the series in (5.1) diverges. Fix $k \neq 0$. Since, for all real $x$,

$$
1-\operatorname{Re} e(x)=1-\cos (2 \pi x) \leq 2 \pi^{2}\|x\|^{2},
$$

we have, for all $j$,

$$
\begin{aligned}
1-\frac{1}{q_{j}}\left|\sum_{0 \leq n<q_{j}} e\left(k f_{j}(n)\right)\right| & \leq 1-\frac{1}{q_{j}} \operatorname{Re} \sum_{0 \leq n<q_{j}} e\left(k f_{j}(n)\right) \\
& =\frac{1}{q_{j}} \sum_{0 \leq n<q_{j}}\left(1-\operatorname{Re} e\left(k f_{j}(n)\right)\right) \\
& \leq \frac{1}{q_{j}} \sum_{0 \leq n<q_{j}} 2 \pi^{2}\left\|k f_{j}(n)\right\|^{2} \\
& \leq \pi^{2} \sum_{0 \leq n<q_{j}}\left\|k f_{j}(n)\right\|^{2} .
\end{aligned}
$$

Thus, condition (ii) of Theorem 1 implies the divergence of the series in (5.1), as claimed.

Proof of Corollary 2. Assume first that $\alpha$ is irrational. If the factors $q_{j}$ are bounded, then, since $f_{j}(1)=\alpha$ for all $j$, it follows from Corollary 1 that $f$ is uniformly distributed modulo 1 . It remains to deal with the case when the factors $q_{j}$ are unbounded.

Fix $k \neq 0$. Then we have, for all $j \geq 0$,

$$
\begin{aligned}
\frac{1}{q_{j}}\left|\sum_{0 \leq n<q_{j}} e\left(k f_{j}(n)\right)\right| & =\frac{1}{q_{j}}\left|\sum_{0 \leq n<q_{j}} e(k \alpha n)\right| \\
& =\frac{1}{q_{j}}\left|\frac{1-e\left(k \alpha q_{j}\right)}{1-e(k \alpha)}\right| \leq \frac{2}{q_{j}(1-e(\alpha k))} .
\end{aligned}
$$

Since the factors $q_{j}$ are unbounded and $\alpha$ is irrational, this quantity is $\leq 1 / 2$ for infinitely many $j$, and so condition (ii) of Theorem 1 is satisfied. Therefore, $f$ has a uniform limit distribution modulo 1 .

On the other hand, if $\alpha$ is rational, then $f$ takes on only finitely many values modulo 1 , and thus $f$ cannot be uniformly distributed modulo 1 .

Proof of Corollary 3. Let $F=\alpha f$. Then $F$ is completely $Q$-additive with component functions $F_{j}=\alpha f_{j}$. As in Corollary 2, if $\alpha$ is rational then $F$ cannot be uniformly distributed modulo 1 . Assume therefore that $\alpha$ is 
irrational. We will show that condition (ii) of Theorem 1 is satisfied (with $F$ in place of $f$ ) for all $k \neq 0$. By Theorem 1 it then follows that $F$ is uniformly distributed modulo 1. Fix an integer $k \neq 0$ and let $a$ be as in the statement of the corollary, so that $f_{0}(a)>0$ and $a<q_{j}$ for all $j$. As in the proof of Corollary 1 , we have, for all $j$,

$$
1-\frac{1}{q_{j}}\left|\sum_{0 \leq n<q_{j}} e\left(k F_{j}(n)\right)\right| \geq \frac{2\left\|k F_{j}(a)\right\|^{2}}{q_{j}}=\frac{2\left\|k \alpha f_{j}(a)\right\|^{2}}{q_{j}}=\frac{2\left\|k \alpha f_{0}(a)\right\|^{2}}{q_{j}} .
$$

Since $\left\|k \alpha f_{0}(a)\right\| \neq 0$ by our assumptions that $\alpha$ is irrational, $k \neq 0$, and $f_{0}(a) \neq 0$, and since, by the hypothesis of Corollary 2 , the series $\sum_{j \geq 0} 1 / q_{j}$ diverges, condition (ii) of Theorem 1 is satisfied as claimed.

Proof of Corollary 4. We note first that the component functions $f_{j}(n)$ of $f(n)=\alpha M(n)$ are given by

$$
f_{j}(n)= \begin{cases}\alpha, & n=q_{j}-1 \\ 0, & 0 \leq n<q_{j}-1\end{cases}
$$

Thus we have, for any integer $k \neq 0$,

$$
\sum_{0 \leq n<q_{j}} e\left(k f_{j}(n)\right)=q_{j}-1+e(k \alpha) .
$$

It follows that condition (i) of Theorem 1 is satisfied if and only if, for some $j, q_{j}=2$ and $\|k \alpha\|=0$. Since $\alpha$ is irrational, this is impossible unless $k=0$. Therefore, condition (i) of Theorem 1 does not hold for any $k \neq 0$.

We next show that condition (ii) of Theorem 1 is equivalent to the divergence of $\sum_{j \geq 0} 1 / q_{j}$. In view of (5.2), condition (ii) of Theorem 1 is equivalent to

$$
\sum_{j \geq 0}\left(1-\frac{1}{q_{j}}\left|q_{j}-1+e(k \alpha)\right|\right)=\infty .
$$

To show the equivalence between (5.3) and the divergence of $\sum_{j \geq 0} 1 / q_{j}$, we will establish the inequalities

$$
\frac{4\|k \alpha\|^{2}}{q} \leq 1-\frac{1}{q}|q-1+e(k \alpha)| \leq \frac{2}{q}
$$

for any integer $q \geq 2$ and any real number $\alpha$.

The upper bound in (5.4) is trivial. To prove the lower bound, we note that

$$
\begin{aligned}
\left(\frac{1}{q}|q-1+e(k \alpha)|\right)^{2} & \leq \frac{1}{q^{2}}\left((q-1)^{2}+1+2(q-1) \cos (2 \pi k \alpha)\right) \\
& =\frac{1}{q^{2}}\left(q^{2}-2(q-1)(1-\cos (2 \pi k \alpha))\right)
\end{aligned}
$$




$$
\begin{aligned}
& =1-\frac{2(q-1)}{q^{2}}(1-\cos (2 \pi k \alpha)) \\
& \leq\left(1-\frac{q-1}{q^{2}}(1-\cos (2 \pi k \alpha))\right)^{2} \\
& \leq\left(1-\frac{(q-1)\left(8\|k \alpha\|^{2}\right)}{q^{2}}\right)^{2} \\
& \leq\left(1-\frac{4\|k \alpha\|^{2}}{q}\right)^{2}
\end{aligned}
$$

It follows that

$$
\frac{1}{q}|q-1+e(k \alpha)| \leq 1-\frac{4\|k \alpha\|^{2}}{q},
$$

which implies the lower bound in (5.4). Since $\alpha$ is irrational, we have $\|k \alpha\| \neq$ 0 for all non-zero integers $k$. Thus condition (ii) of Theorem 1 holds for all $k \neq 0$ if and only if $\sum_{j \geq 0} 1 / q_{j}$ diverges. From the theorem it therefore follows that $f$ is uniformly distributed modulo 1 if and only if $\sum_{j \geq 0} 1 / q_{j}$ diverges.

It remains to show that $f$ has a non-uniform limit distribution modulo 1 if and only if the series $\sum_{j>0} 1 / q_{j}$ converges. To this end we note that, by (5.2), the first part of condition (iii) of Theorem 1 is equivalent to the convergence of

$$
\sum_{j \geq 0} \frac{1-e(k \alpha)}{q_{j}}
$$

which in turn is equivalent to the convergence of $\sum_{j \geq 0} 1 / q_{j}$, since $\alpha$ is irrational. Therefore it remains only to show that if $\sum_{j \geq 0} 1 / q_{j}$ converges, then the second part of condition (iii) of Theorem 2.1 holds for all $k \neq 0$. This follows immediately from the observation that, for all $k \neq 0$,

$$
\max _{0<n \leq q_{j}} \frac{1}{n} \sum_{0 \leq m<n}\left\|k f_{j}(m)\right\|^{2}=\frac{\|k \alpha\|^{2}}{q_{j}} \rightarrow 0,
$$

as $j$ tends to infinity, since the convergence of $\sum_{j \geq 0} 1 / q_{j}$ implies that $1 / q_{j}$ tends to 0 .

Proof of Corollary 5. We note first that, for all $j$ with $q_{j}>a$,

$$
f_{j}(n)= \begin{cases}\alpha, & n=a \\ 0, & \text { otherwise. }\end{cases}
$$

Thus, we have

$$
\sum_{0 \leq n<q_{j}} e\left(k f_{j}(n)\right)= \begin{cases}q_{j}-1+e(k \alpha), & q_{j}>a \\ q_{j}, & q_{j} \leq a\end{cases}
$$


As in the proof of Corollary 4, this implies that condition (i) of Theorem 1 does not hold for any $k \neq 0$. Moreover, using (5.4), we see that condition (ii) of Theorem 1 is satisfied for all $k \neq 0$ if and only if $\sum_{q_{j}>a} 1 / q_{j}$ diverges. Therefore, $f$ is uniformly distributed modulo 1 if and only if $\sum_{q_{j}>a} 1 / q_{j}$ diverges. This proves the first assertion of the corollary.

To prove the second assertion of the corollary, we note that by (5.5), we have, for all $k \neq 0$,

$$
\max _{0<n \leq q_{j}} \frac{1}{n} \sum_{0 \leq m<n}\left\|k f_{j}(m)\right\|^{2}= \begin{cases}\|k \alpha\|^{2} / a, & q_{j}>a, \\ 0, & q_{j} \leq a .\end{cases}
$$

Therefore, the limit in condition (iii) of Theorem 1 is 0 for all $k \neq 0$ if and only if $q_{j} \leq a$ for all but at most finitely many $j$. It remains only to show that under the same condition, the series in condition (iii) of Theorem 1 converges for all $k \neq 0$. This follows immediately, since, by (5.6),

$$
\sum_{j \geq 0}\left(1-\frac{1}{q_{j}} \sum_{0 \leq n<q_{j}} e\left(k f_{j}(n)\right)\right)=\sum_{q_{j}>a} \frac{1-e(k \alpha)}{q_{j}} .
$$

Acknowledgments. The author wishes to gratefully acknowledge the advice and encouragement provided by Professor A. J. Hildebrand in the preparation of this paper.

\section{References}

[BG] G. Barat and P. Grabner, Distribution properties of G-additive functions, J. Number Theory 60 (1996), 103-123.

[Co1] J. Coquet, Remarques sur les nombres de Pisot-Vijayaraghavan, Acta Arith. 32 (1977), 79-87.

[Co2] -, Sur certaines suites uniformément équiréparties modulo 1, ibid. 36 (1980), $157-162$.

[De1] H. Delange, Sur les fonctions arithmétiques multiplicatives, Ann. Sci. École Norm. Sup. (3) 78 (1961), 273-304.

[De2] - On the distribution modulo 1 of additive functions, J. Indian Math. Soc. 34 (1970), 215-235.

[De3] -, Sur les fonctions q-additives ou q-multiplicatives, Acta Arith. 21 (1972), 285298.

[DT] M. Drmota and R. F. Tichy, Sequences, Discrepancies and Applications, Lecture Notes in Math. 1651, Springer, Berlin, 1997.

[El] P. D. T. A. Elliott, Probabilistic Number Theory, Vol. 1, Springer, New York, 1979 .

[Fr] A. Fraenkel, Systems of numeration, Amer. Math. Monthly 92 (1985), 105-114.

[Ge] A. O. Gel'fond, Sur les nombres qui ont des propriétés additives et multiplicatives données, Acta Arith. 13 (1967/1968), 259-265.

[GLT] P. J. Grabner, P. Liardet and R. F. Tichy, Odometers and systems of numeration, ibid. 70 (1995), 103-123. 
[Ho] A. Hoit, The distribution of generalized sum-of-digits functions in residue classes, J. Number Theory 79 (1999), 194-216.

[Ki] D.-H. Kim, Topics in the theory of q-additive functions and q-multiplicative functions, PhD thesis, Univ. of Illinois at Urbana-Champaign, 1998.

[KT] P. Kirschenhofer and R. F. Tichy, On the distribution of digits in Cantor representations of integers, J. Number Theory 18 (1984), 121-134.

[LR] N. Levinson and R. Redheffer, Complex Variables, Holden-Day, Inc., California, 1970.

[Ma] E. Manstavičius, Probabilistic theory of additive functions related to systems of numeration, in: New Trends in Probability and Statistics, Vol. 4, VSP, Palanga, 1997, 413-429.

[MF] M. Mendès France, Deux remarques concernant l'équirépartition des suites, Acta Arith. 14 (1967/1968), 163-167.

[We] H. Weyl, Über die Gleichverteilung von Zahlen mod Eins, Math. Ann. 77 (1916), 313-352.

Department of Mathematics

Elmhurst College

Elmhurst, IL 60126, U.S.A.

E-mail: abigailh@elmhurst.edu

Received on 19.7.1999

and in revised form on 11.4.2001 